\title{
Avaliação da administração de coenzima Q10 na atenuação de respostas oxidativas da agregação $\beta$-Amiloide em modelos de doença de Alzheimer: Uma revisão
}

\section{sistemática de literatura}

\author{
Evaluation of coenzyme Q10 administration in attenuating oxidative responses of $\beta$-Amyloid \\ aggregation in models of Alzheimer's disease: A systematic literature review \\ Evaluación de la administración de coenzima Q10 para atenuar las respuestas oxidativas de la \\ agregación $\beta$-amiloide en modelos de enfermedad de Alzheimer: Revisión sistemática de la

Francisco Emanoel Alves de Araújo ORCID: https://orcid.org/0000-0002-5020-172X Universidade Potiguar, Brasil

E-mail: franciscoemanoel6341@gmail.com João Matheus Caé da Rocha ORCID: https://orcid.org/0000-0002-4109-4598 Universidade Potiguar, Brasil E-mail: j.matheus6@gmail.com

Bruna Jéssica Dantas de Lucena ORCID: https://orcid.org/0000-0003-0526-3347 Universidade Potiguar, Brasil

E-mail: brunalucena@outlook.com.br

Sarah Vitória Gomes de Sousa ORCID: https://orcid.org/0000-0003-3830-9209 Universidade Potiguar, Brasil E-mail: sv453141@gmail.com

Maria Raquel Araújo de Sousa ORCID: https://orcid.org/0000-0003-1700-8386 Universidade Potiguar, Brasil

E-mail: mariaraquelsda@gmail.com

Lucas Emmanuel Rocha de Moura Marques ORCID: https://orcid.org/0000-0002-8004-8121 Universidade Potiguar, Brasil

E-mail: lucasmarques071@ @otmail.com João Lindemberg Bandeira de Lima ORCID: https://orcid.org/0000-0001-8526-474X Universidade Potiguar, Brasil E-mail: bblcrist@hotmail.com

Kellyson Lopes da Silva Macedo ORCID: https://orcid.org/0000-0003-0574-8512 Universidade Potiguar, Brasil

E-mail: kellysonlopessilva@gmail.com

Salvador Viana Gomes Junior ORCID: https://orcid.org/0000-0002-5173-8318 Universidade Potiguar, Brasil E-mail: salvajunior@live.com

\begin{abstract}
Resumo
O objetivo deste estudo foi avaliar os efeitos da administração de CoQ10 na atenuação de respostas oxidativas por agregação $\beta$-Amiloide em modelos de doença de Alzheimer. O presente trabalho trata-se de uma revisão sistemática da literatura, constituída de etapas estabelecidas por estratégias de busca, identificação, seleção e elegibilidade de estudos. A CoQ10 ou ubi-quinona e ubidecarenona, é uma pró-vitamina sintetizada de forma endógena, esta tem sido utilizada em ensaios clínicos para avaliar seu potencial de neuroproteção e antioxidação. Os achados mostraram que a CoQ10 apresenta efeitos antioxidantes e de diminuição da deposição intracelular do composto beta-amilóide, exercendo o alívio oxidativo e efeito antiapoptótico. A administração de CoQ10 demonstrou resultados significativos na atenuação de respostas oxidativas de agregação $\beta$-Amiloide em modelos da DA.
\end{abstract}


Palavras-chave: Doença de Alzheimer; Estresse oxidativo; Coenzima Q10.

\begin{abstract}
The aim of this study was to evaluate the effects of CoQ10 administration on attenuation of oxidative responses by $\beta$-Amyloid aggregation in models of Alzheimer's disease. The present work is a systematic review of the literature, consisting of steps established by search strategies, identification, selection and eligibility of studies. CoQ10 or ubiquinone and ubidecarenone, is a pro-vitamin synthesized in an endogenous way, this has been used in clinical trials to evaluate its potential for neuroprotection and antioxidation. The findings showed that CoQ10 has antioxidant effects and decreases the intracellular deposition of the beta-amyloid compound, exerting oxidative relief and antiapoptotic effect. The administration of CoQ10 demonstrated significant results in the attenuation of oxidative responses of $\beta$-Amyloid aggregation in AD models.
\end{abstract}

Keywords: Alzheimer's disease; Oxidative stress; Coenzyme Q10.

\title{
Resumen
}

El objetivo de este estudio fue evaluar los efectos de la administración de CoQ10 sobre la atenuación de las respuestas oxidativas por la agregación de $\beta$-amiloide en modelos de la enfermedad de Alzheimer. El presente trabajo es una revisión sistemática de la literatura, consistente en pasos establecidos por estrategias de búsqueda, identificación, selección y elegibilidad de estudios. CoQ10 o ubi-quinona y ubidecarenona, es una provitamina sintetizada de forma endógena, esta ha sido utilizada en ensayos clínicos para evaluar su potencial de neuroprotección y antioxidación. Los hallazgos mostraron que la CoQ10 tiene efectos antioxidantes y disminuye la deposición intracelular del compuesto beta-amiloide, ejerciendo un alivio oxidativo y un efecto antiapoptótico. La administración de CoQ10 demostró resultados significativos en la atenuación de las respuestas oxidativas de la agregación de $\beta$-amiloide en modelos de EA.

Palabras clave: Enfermedad de Alzheimer; Estrés oxidativo; Coenzima Q10.

\section{Introdução}

A doença de Alzheimer (DA) é caracterizada pela presença de placas senis formadas por depósitos fibrilares insolúveis de peptídeo beta-amilóide no cérebro. No envelhecimento e particularmente na DA, esse peptídeo se acumula, favorecendo a formação de fibrilas insolúveis e causando neurotoxicidade. A toxicidade do peptídeo beta-amilóide tem sido associada à geração de radicais livres que, por sua vez, promovem a peroxidação lipídica e a oxidação de proteínas (ManzanoLeón \& Mas-Oliva, 2006). Algumas espécies de radicais livres medeiam danos a proteínas, lipídios, mitocôndrias e DNA e podem ativar o ciclo celular, sobrecarregar as defesas antioxidantes endógenas do cérebro, e ainda contribuir para danos em neurônios (Lovell \& Markesbery, 2007), sendo assim, o estresse oxidativo (EO) participa do desenvolvimento da DA promovendo a deposição de A $\beta$, hiperfosforilação de tau, a perda de sinapses e de neurônios (Chen \& Zhong, 2014). Suas principais manifestações clínicas são a perda progressiva de memória e linguagem, distúrbios psiquiátricos e comportamentais, e prejuízos no desempenho das atividades da vida cotidiana (Farlow, 1998). Seu diagnóstico clínico é baseado e norteado por uma vasta avaliação que podem incluir o histórico do paciente, avaliação cognitiva e funcional, e investigações, guiadas por critérios diagnósticos aceitos (Eratne et al., 2018).

De acordo com relatórios fornecidos pela Organização Mundial de Saúde (OMS), cerca de 60\% a 70\% das demências é representada pela DA, dado este colhido diante do Global Dementia Observatory, plataforma que oferece dados sobre demências e suas epidemiologias e incidências, demonstrando assim, a DA como a demência de maior prevalência no mundo (OMS, 2020).

Dessa maneira, nos últimos anos, os antioxidantes têm sido sugeridos como agentes possivelmente úteis para a prevenção e tratamento da DA (Darvesh et al., 2010), já que por sua vez eles têm sido associados na redução de atividade deletéria das espécies reativas de oxigênio (ROS) (Manzano-León \& Mas-Oliva, 2006).

A coenzima Q10 (CoQ10) também conhecida como ubi-quinona e ubidecarenona, é uma pró-vitamina sintetizada endogenamente e envolvida em uma variedade de processos celulares, mais notavelmente na cadeia de transporte de elétrons mitocondrial e na síntese de trifosfato de adenosina (ATP) (Lockwood et al., 1994). Sendo assim, a CoQ10 tem sido usada como suplemento dietético ou medicamento há mais de 30 anos. Dados publicados sobre sua segurança em estudos pré- 
clínicos e clínicos demonstraram que CoQ10 não causa efeitos adversos graves em humanos, e é seguro para uso como suplemento dietético (Hidaka et al., 2008). O Ácido para-hidroxibenzoico do aminoácido tirosina é o primeiro precursor aromático na via biossintética da CoQ10 em humanos, e constitui a estrutura do anel quinóide da molécula de CoQ10. A cauda, que consiste em 10 unidades isoprenóides, é derivada da via do mevalonato (Folkers, 1996). Além disso, a CoQ10 apresenta como cascata de mecanismo de ação o envolvimento na síntese de trifosfato de adenosina (ATP), afetando a função de todas as células do corpo, especialmente aquelas que apresentam alta demanda energética, tornando-se essencial para a saúde de todos os tecidos e órgãos, demonstrando eficácia significativa na prevenção da oxidação de proteínas, lipídios e do DNA (Littarru \& Tiano, 2010). A biodisponibilidade em suplementos de CoQ10 em humanos parecem depender dos excipientes constituintes das formulações, além das características fisiológicas dos indivíduos (López-Lluch et al., 2019). Isto torna-se imprescindível, uma vez que os benefícios de boas respostas fisiológicas da CoQ10 dependem de sua biodisponibilidade, além de sua captação pelos tecidos, fígado, tecido adiposo e células circulantes (Díaz-Casado et al., 2019).

Os níveis de CoQ10 diminuem com o avanço da idade, e este declínio pode contribuir em parte para algumas das manifestações do envelhecimento (Sohal, \& Forster, 2007). Outros estudos mostraram que a CoQ10 tem ação neuroprotetora na DA através da proteção do dano oxidativo e na atenuação da disfunção mitocondrial (Lee et al., 2009). Além disso, há relatos de resultados ainda exploratórios em relação a DA, onde alguns destes estudos anteriores demonstram efeitos potenciais da CoQ10 sobre tal neuropatologia (Huang et al., 2003).

Com base nisso, o objetivo do estudo de revisão sistemática de literatura foi desenvolvido com o intuito de avaliar os efeitos da administração de CoQ10 na atenuação de respostas oxidativas por agregação $\beta$-Amiloide em modelos de doença de Alzheimer.

\section{Metodologia}

De acordo com Pereira et al (2018), os métodos qualitativos são fundamentados no processo de interpretação pelo pesquisador com suas opiniões sobre o fenômeno estudado. Foi realizada uma pesquisa qualitativa para delineamento de um estudo de revisão sistemática da literatura, elaborada de acordo com a utilização de palavras-chave em recursos de bases de dados científicos disponíveis, para obtenção dos resultados e em subsequência, o delineamento do estudo proposto. Diante disto, foi obtido as seguintes nomenclaturas com registro nos Descritores em Ciências da Saúde (DeCS) para obtenção dos estudos. Os descritores selecionados para filtragem foram: Alzheimer Disease, Amyloid Beta-Peptides, Plaque Amyloid, Oxidative Stress e Ubiquinone. Em seguida, foi utilizado como estratégia de busca "and" entre as palavras para filtragem dos estudos nas bases de dados seguindo o seguinte modelo metodológico: Alzheimer Disease and Ubiquinone, Amyloid BetaPeptides and Ubiquinone, Plaque Amyloid and Ubiquinone, Oxidative Stresse and Alzheimer Disease and Ubiquinone e Oxidative Stresse and Plaque Amyloid and Ubiquinone.

Os estudos foram buscados nas bases de dados selecionadas: Science Direct, National Library of Medicine dos Estados Unidos (PubMed), Latino-Americana de informação bibliográfica em ciências da saúde (LILACS), Web of Science, Scientific Electronic Library Online (SciELO), Biblioteca Cochrane e Elsevier's Scopus. Sendo assim, esta seleção inicial dos estudos foi tomada por base na avaliação dos títulos e resumos, com procura por estudos que relacionaram a utilização da coenzima CoQ10 administrados em modelos da DA. Sendo assim, foram incluídos artigos que realizaram a análise de efeitos da administração de CoQ10 na atenuação de respostas oxidativas de agregação $\beta$-Amiloide em modelos de DA.

No delineamento metodológico do estudo, não foi realizado restrições com relação aos anos de publicação devido a presença de quantidade não proporcional a estudos inclusos nos critérios de inclusão e exclusão. Além disso, não foi realizado restrição quanto ao idioma, contudo os estudos analisados foram apenas aqueles publicados em inglês. Com base nisso, foi definido como critérios de inclusão: Estudos experimentais com uso de coenzima CoQ10, demonstrando os efeitos de sua 
administração na atenuação de respostas especificamente oxidativas da $\beta$-Amiloide em modelos celulares de DA; Todos os modelos utilizados nos estudos devem apresentar os sinais ou sintomas clínicos relacionados a DA. Além disso, foram adotados os seguintes critérios de exclusão: Artigos de revisão; Livros; A coenzima CoQ10 associada a outras suplementações fitoterápicas, medicamentos ou terapia.

O processo seletivo de escolha dos estudos foi realizado com enfoque fundamentado no título e no resumo, e logo após, a publicação foi revisada na íntegra. Não foram considerados consociação de dados estatísticos neste estudo devido a ampla diversidade metodológica entre os estudos, pois havia diversas variações quanto aos métodos de avaliação e as doses administradas. Portanto, as meta-análises não foram aplicadas com base nos estudos e dados avaliados.

\section{Resultados e Discussão}

Por conseguinte, a seleção inicial obteve um total de 1481 estudos encontrados (865 no Science Direct, 438 no PubMed, 23 na LILACS, 42 na Web of Science, 21 na SciELO, 54 na Biblioteca Cochrane e 38 na Elsevier's Scopus). Destes estudos, 1133 foram excluídos por duplicidade entre as bases de dados, 5 por serem livros e 1128 por serem artigos de revisão. Dos 348 estudos restantes, 227 foram excluídos após uma avaliação do título. O próximo passo foi ler os resumos. Após a aplicação dos critérios de exclusão e inclusão mencionados, um total de 58 artigos foram excluídos. Portanto, 63 artigos foram revisados na íntegra, dos quais 56 foram excluídos por não se enquadrarem em nossos critérios de inclusão e exclusão, pois grande parte desses estudos eram comparativos da CoQ10 com outras suplementações fitoterápicas, medicamentos ou terapia, ou com estudo experimental realizado em modelos diferente do proposto. Finalmente, um total de 7 artigos foram incluídos nesta revisão. O fluxograma dos estudos selecionados é mostrado na Figura 1 a seguir. 
Research, Society and Development, v. 10, n. 2, e41210212751, 2021

(CC BY 4.0) | ISSN 2525-3409 | DOI: http://dx.doi.org/10.33448/rsd-v10i2.12751

Figura 1. Fluxograma do processo metodológico de triagem dos artigos.

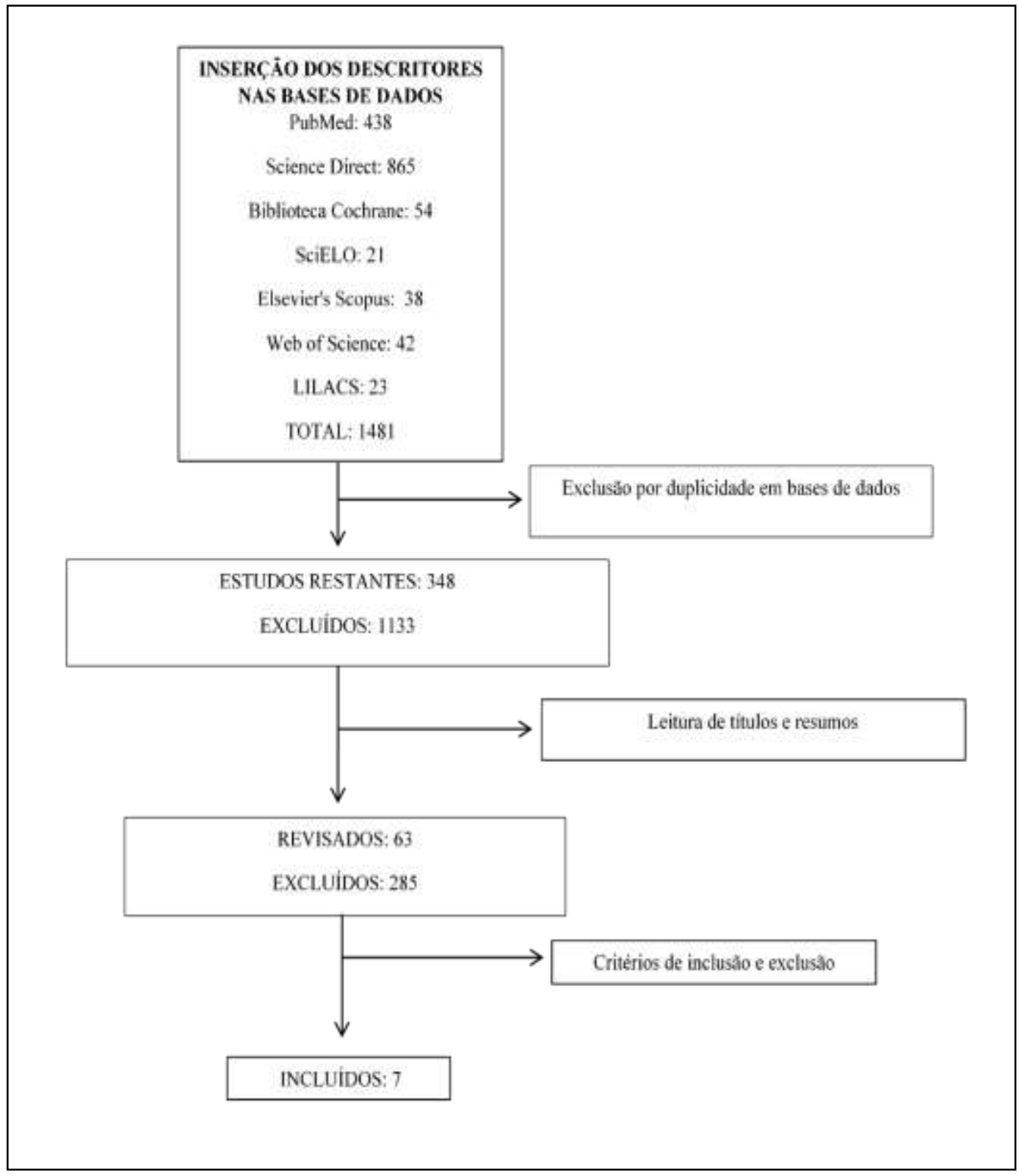

Fonte: Autores.

Com base nos resultados de busca e leitura dos estudos, foram selecionados 7 que se encaixaram ao tema proposto e os critérios de inclusão, e que avaliaram a administração de CoQ10 na atenuação de respostas oxidativas de agregação $\beta$ Amiloide em modelos de DA. Sendo assim, os resultados dos estudos foram descritos na Tabela 1 a seguir. 
Tabela 1. Tabela de contendo resultados dos estudos sobre CoQ10.

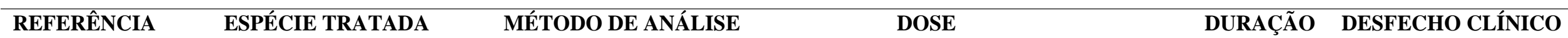

\begin{tabular}{|c|c|c|c|c|c|c|}
\hline $\begin{array}{l}\text { YANG } \\
\text { colaboradores } \\
(2010)\end{array}$ & e & $\begin{array}{l}\text { Cérebro de camundongos } \\
\text { com grupo controle }(n=5) \text { e } \\
\text { grupo de tratamento }(n=4)\end{array}$ & $\begin{array}{l}\text { Imunohistoquímica e análise de imagem } \\
\text { por ressonância magnética }(\mathrm{RM}) \text {. }\end{array}$ & $\begin{array}{l}\text { Administração oral de CoQ10 } \\
(1.200 \mathrm{mg} / \mathrm{kg} / \text { dia })\end{array}$ & 60 dias & $\begin{array}{l}\text { Ação antioxidante e } \\
\text { significativa da de } \\
\text { intracelular beta-amilóição } \\
\text { inder }\end{array}$ \\
\hline $\begin{array}{l}\text { DURÁN-PRADO } \\
\text { colaboradores } \\
(\mathbf{2 0 1 4})\end{array}$ & $\mathbf{e}$ & $\begin{array}{l}\text { Células endoteliais da veia } \\
\text { umbilical } \\
\text { (HUVECs) }\end{array}$ & $\begin{array}{l}\text { Microscopia de fluorescência e confocal, } \\
\text { técnicas bioquímicas e metabolômica } \\
\text { baseada em RMN. }\end{array}$ & $5 \mu \mathrm{M}$ de CoQ10 & 24 horas & $\begin{array}{l}\text { Alivio de lesão oxidativa induzida } \\
\text { por } \mathrm{A} \beta, \quad \text { antinecrótico } \\
\text { antiapoptótico. }\end{array}$ \\
\hline $\begin{array}{l}\text { YANG } \\
\text { colaboradores } \\
(2008)\end{array}$ & e & $\begin{array}{l}\text { Camundongos selvagens não } \\
\text { tratado }(\mathrm{n}=\quad 11), \\
\text { camundongos transgênicos } \\
\text { não tratados compreendendo } \\
\text { L235P PS-1 mutante ( } \mathrm{n}= \\
\text { 10); Camundongos } \\
\text { transgênicos tratados com } \\
\text { CoQ10 (n = 10). Houve a } \\
\text { remoção de cérebro. }\end{array}$ & $\begin{array}{l}\text { Imunofluorescência e coloração com } \\
\text { vermelho do } \text { Congo, ensaio } \\
\text { imunoenzimático, nível elevado } \\
\text { demalondialdeído (MDA) e diminuição } \\
\text { da atividade de superóxido dismutase } \\
\text { (SOD). }\end{array}$ & CoQ10 (1.200 mg kg/1dia) & 60 dias & $\begin{array}{l}\text { Ação antioxidante e redução } \\
\text { significativa nos níveis de produção } \\
\text { e acúmulo de } \mathrm{A} \beta 42 \text {. }\end{array}$ \\
\hline $\begin{array}{l}\text { CHOI } \\
\text { colaboradores } \\
(\mathbf{2 0 1 2})\end{array}$ & $\mathbf{e}$ & Neurônios corticais de ratos & $\begin{array}{l}\text { Incubação com fluorescentesonda } 2{ }_{0}, 70^{-} \\
\text {diclorodihidrofluoresceína } \\
\text { (DCFH-DA). }\end{array}$ & $\begin{array}{l}\text { Tratados com várias } \\
\text { concentrações de CoQ10 (0, } \\
0,01,0,1,1,10 \text { ou } 100 \mathrm{~m} \mathrm{M})\end{array}$ & 48 horas & $\begin{array}{l}\text { Ação antioxidante pela inibição de } \\
\text { EO. }\end{array}$ \\
\hline $\begin{array}{l}\text { WADSWORTH } \\
\text { colaboradores } \\
(\mathbf{2 0 0 8 )}\end{array}$ & e & $\begin{array}{l}\text { Células de neuroblastoma } \\
\text { MC65. Os camundongos } \\
\text { foram randomizados para } \\
\text { receber dieta controle (AIN- } \\
\text { 93M, Dyets Inc. Bethlehem, } \\
\text { PA), AIN-93M } \\
\text { suplementado com CoQ } \\
\text { (Vitaline }{ }^{\circledR} \text { Formulas, Green } \\
\text { Bay, WI) ou lovastatina }\end{array}$ & $\begin{array}{l}\text { Ensaio BCA (Pierce Biotechnology, } \\
\text { Rockford, IL). Carbonilos de proteína } \\
\text { foram determinados por ELISA } \\
\text { colorimétrico usando o Kit de Ensaio de } \\
\text { Enzima de Carbonil de Proteína Biocell } \\
\text { (Biocell Corp, Aukland, NZ). Os níveis } \\
\text { de malonaldeído (MDA) foram medidos } \\
\text { como um indicador de peroxidação } \\
\text { lipídica usando o Ensaio Bioxytevch LPO }\end{array}$ & $10 \mathrm{~g} / \mathrm{kg}$ & 30 dias & $\begin{array}{l}\text { Efeitos antioxidantes in vitro e no } \\
\text { cérebro de camundongos. }\end{array}$ \\
\hline
\end{tabular}




\begin{tabular}{|c|c|c|c|c|c|c|}
\hline & & $\begin{array}{l}\text { (Axxora LLC, San Diego, } \\
\text { CA) com uma dose de } 2 \mathrm{~g} / \\
\text { kg de dieta. }\end{array}$ & 586 (Oxis International). & & & \\
\hline $\begin{array}{l}\text { KOMAKI } \\
\text { colaboradores } \\
\text { (2019) }\end{array}$ & e & $\begin{array}{l}50 \text { ratos Wistar machos } \\
\text { adultos divididos em cinco } \\
\text { grupos: grupo controle } \\
\text { (salina); grupo sham; injeção } \\
\text { intraventricular de PBS, } \\
\text { grupo A } \beta \text { injeção } \\
\text { intraventricular de A } \beta \text {, grupo } \\
\text { Q10; e Q10 via gavagem oral } \\
\text { e grupo Q10+ A } \beta \text {. }\end{array}$ & $\begin{array}{l}\text { Registros eletrofisiológicos in vivo, } \\
\text { indução LTP e análises bioquímicas. }\end{array}$ & $\begin{array}{l}\text { Grupo } 4 \text { (Q10; recebeu } 50 \mathrm{mg} / \\
\mathrm{kg} \text { de Q10 por gavagem oral } \\
\text { uma vez ao dia), e grupo 5 (Q10 } \\
+\quad \mathrm{A} \beta \text {; recebeu Q10 por } \\
\text { gavagem oral uma vez ao dia e } \\
\text { injeções intraventriculares de } \\
\mathrm{A} \beta \text { ). }\end{array}$ & 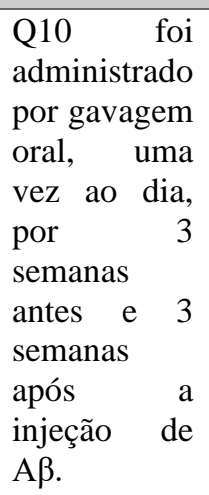 & $\begin{array}{l}\text { Antioxidante } \quad \text { e antiapoptótica. } \\
\text { Redução dos efeitos de } A \beta \text { e } \\
\text { neuroproteção contra os efeitos } \\
\text { prejudiciais do } A \beta \text { na plasticidade } \\
\text { sináptica do hipocampo. }\end{array}$ \\
\hline $\begin{array}{l}\text { DUMONT } \\
\text { colaboradores } \\
\text { (2011) }\end{array}$ & e & $\begin{array}{l}\text { Células de neuroblastoma de } \\
\text { camundongo carregando } \\
\text { AßPP com a mutação } \\
\text { KM670 / 671NL. }\end{array}$ & $\begin{array}{l}\text { Imunohistoquímica e quantificação, } \\
\text { Western blotting, Sandwish ELISA de } \\
\text { peptídeos A } \beta \text { 42. }\end{array}$ & $\begin{array}{l}\text { O primeiro coorte de } \\
\text { camundongos foi tratada com } \\
0,4 \% \text { de CoQ10 em ração ou } \\
\text { ração controle. O segundo } \\
\text { coorte de camundongos foi } \\
\text { tratada com } 2,4 \% \text { de CoQ10 em } \\
\text { ração ou ração controle. }\end{array}$ & $\begin{array}{l}\text { Primeiro } \\
\text { coorte com } \\
\text { duração de } 3 \\
\text { meses, e } \\
\text { segundo } \\
\text { coorte com } \\
\text { duração de } 5 \\
\text { meses. }\end{array}$ & $\begin{array}{l}\text { Efeito antioxidante pela diminuição } \\
\text { significativa de EO dos níveis } \\
\text { cerebrais de carbonilas de proteínas, } \\
\text { um marcador de EO e redução dos } \\
\text { níveis de A } \beta \text { 42. Além disso, } \\
\text { apresentou melhoria de desempenho } \\
\text { cognitivo. }\end{array}$ \\
\hline
\end{tabular}

Fonte: Autores. 
A DA se constitui por um distúrbio neurodegenerativo, sendo caracterizada pela deposição de placas beta-amilóide (A $\beta$ ), tau hiperfosforilada (uma proteína que se liga aos microtúbulos) e emaranhados neurofibrilares (NFTs) (Gustafson et al., 2007). O comprometimento no metabolismo da proteína precursora de amiloide (APP) pode levar ao aumento da produção de $A \beta$. Níveis de produção da $A \beta$ aumentados se correlacionam com algumas eventualidades críticas, tais como a formação de NFTs, perda de neurônios, perda de sinapse e disfunção de neurotransmissão (Wang et al., 2012), sendo estas consideradas alterações ao qual podem estar envolvidas em processos de ativação do receptor do N-metil-D-aspartato (NMDA) e ao EO. Além disso, também há relatos de que a $\mathrm{A} \beta$ está incluída em processos de desencadeamento do influxo de Ca $2+$ mediado pela NMDA, danos ou morte neuronal e vias de sinalização relacionadas ao estresse em neurônios que podem exacerbar aumentos relacionados ao envelhecimento no EO, metabolismo energético prejudicado e homeostase de Ca 2+ defeituosa (Bezprozvanny \& Mattson, 2008).

Os receptores N-metil-D-aspartato (NMDARs) são canais catiônicos controlados por intermédio de neurotransmissores inibitórios denominados de glutamato, que apresentam ações críticas no processo de plasticidade, transmissão sináptica excitatória, e em eventos de excitotoxicidade no sistema nervoso central (SNC). A ativação da liberação de glutamato em NMDAR leva a grandes fluxos de Ca 2+ para as células pós sinápticas, ao qual há propostas de que o influxo de $\mathrm{Ca} 2+$ induzido por $\mathrm{A} \beta$ oligomérico intercorre através de NMDAR pós-sináptico. Além disso, esta operação pode conduzir à formação excessiva de ROS e EO (De Felice et al., 2007).

Kamat et al. (2013) provaram que a ativação da NMDAR, fluxos excessivos de Ca 2+ e a geração de radicais livres estão associados com a disfunção sináptica e fosforilação de tau. Com base nisso, se analisa a ampla complexidade da relação entre o EO e a DA (Cioffi et al., 2019).

Figura 2. Comprometimento no metabolismo da proteína precursora de amiloide (APP).

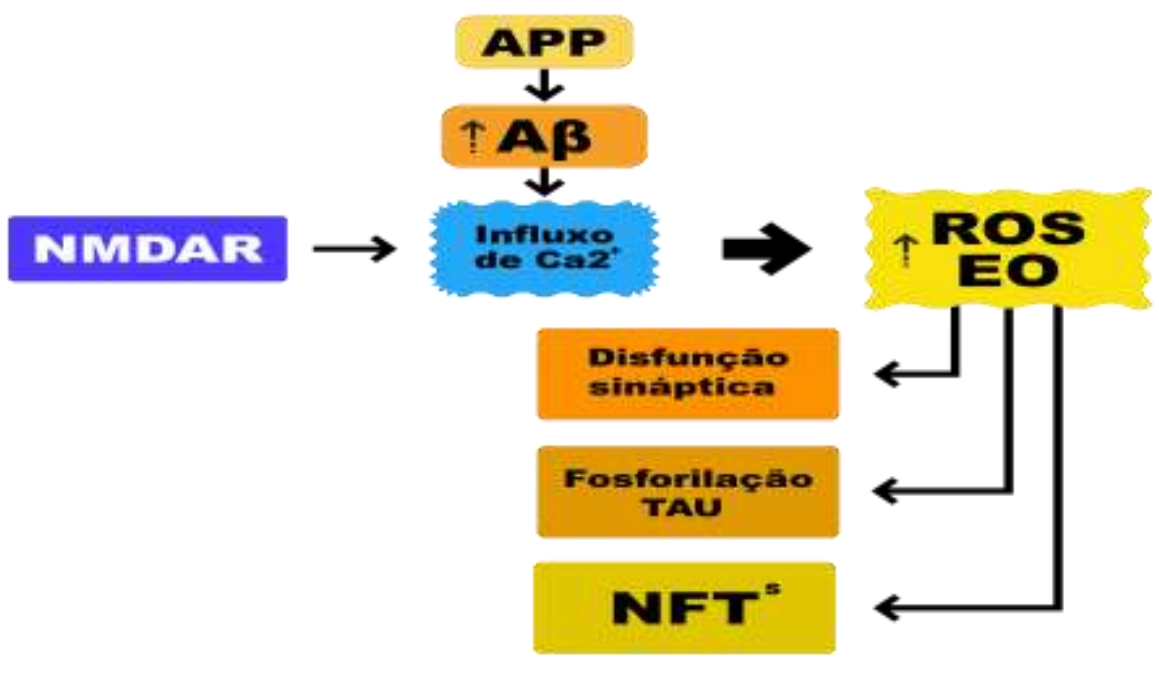

O comprometimento no metabolismo da proteína precursora de amiloide (APP) pode levar ao aumento da produção de A $\beta$. A A $\beta$ com seus níveis de produção aumentados está envolvida nos processos de desencadeamento de influxo de Ca $2+$ mediado pela NMDA. Este processo metabólico pode conduzir a formação excessiva de ROS e ao EO, que associados podem conduzir a disfunção sináptica, fosforilação de tau e a NFTs. Fonte: Autores. 
As sinapses são conexões entre dois neurônios que permitem a emissão de sinais de informações entre eles. Na maioria das doenças neurodegenerativas, este canal é afetado, podendo levar ao quadro de danificação ou perda. Á vista disso, o acúmulo de evidências científicas traz à tona a sugestão de que a disfunção e a perda das conexões sinápticas podem ser um evento inicial relacionado a progressão da DA, sendo este tipo de degeneração caracterizada pelo agravamento da função cognitiva, perda de sinapses e morte de células neuronais (Rai et al., 2014). Um estudo envolvendo modelos de camundongos transgênicos para avaliação da função sináptica e da plasticidade demonstraram que a transmissão sináptica anormal e potenciação de longo prazo prejudicada (LTP), são frequentemente bem associados com a formação de placa A $\beta$ (Roberson et al., 2011).

Uma larga quantidade das terapias usadas na DA passou por ensaios clínicos, entretanto os resultados não foram tão bem-sucedidos até o presente momento. Dessa maneira, lacunas significativas são necessárias para compreender e melhorar as terapias atuais (Kamat et al., 2016). Nos últimos anos, os antioxidantes têm sido sugeridos como possíveis agentes terapêuticos úteis para a prevenção e tratamento da DA (Darvesh et al., 2010). A CoQ10 é um antioxidante natural que ajuda a proteger as mitocôndrias de ROS geradas durante o metabolismo oxidativo (Beal, 2004). A CoQ10 é amplamente utilizada como um componente essencial na geração de ATP no processo de fosforilação oxidativa, e como antioxidante, prevenindo assim, a peroxidação lipídica e eliminando o superóxido (Choi et al., 2005).

Com relação a eficácia em sua administração, no estudo de Durán-Prado et al. (2014) foi administrado $5 \mu \mathrm{M}$ de CoQ10 em células endoteliais da veia umbilical humana (HUVECs) num período de 24 horas. Os resultados demonstraram que a CoQ10 inibiu significativamente a captação e o tráfego mitocondrial do peptídeo A $\beta$ nas células endoteliais humanas, trazendo alívio de lesão oxidativa induzida por $\mathrm{A} \beta$, efeito antinecrótico e antiapoptótico. Outro estudo com uso de pequenas concentrações de dosagem de $(0,0,01,0,1,1,10$ ou $100 \mathrm{~m} \mathrm{M})$ por 48 horas em neurônios corticais de ratos também demonstraram ação antioxidante significativa pela inibição de EO. A CoQ10 reduziu os níveis de radicais livres de maneira dependente da dose. Enquanto isso, o tratamento com CoQ10 pode aumentar os níveis de expressão de p85aPI3K, Akt fosforilada, glicogênio sintase quinase-3 $\beta$ fosforilada e fator de transcrição de choque térmico, sendo estas proteínas, envolvidas no processo de sobrevivência celular neuronal. Além disso, houve diminuição nos níveis de citocromo citosólico e caspase-3 clivada, que estão associadas à morte de células neuronais. Associado a esses resultados, o estudo sugere que os efeitos neuroprotetores da CoQ10 na neurotoxicidade em A $325-35$ é mediada pela inibição do EO com a ativação da via PI3-K / Akt (Choi et al., 2012).

Segundo o estudo realizado por Choi et al. (2012), a CoQ10 apresentou um alto potencial neuroprotetor em neurônios corticais de ratos, assim como, desempenhou um papel antioxidante após a inibição do EO. Em contrapartida, a pesquisa realizada por Lee et al. (2009) destacou que a disfunção mitocondrial prévia é uma característica observada na DA, e de acordo com os estudos utilizados sobre a administração da CoQ10, é relatado que ela é capaz de elevar a concentração mitocrondrial no cérebro, gerando uma resposta favorável ao EO, proporcionando assim, a ação neuroprotetora.

No estudo de Komaki et al. (2019) realizado em 50 ratos Wistar machos adultos divididos em cinco grupos, o grupo 4 (CoQ10; recebeu $50 \mathrm{mg} / \mathrm{kg}$ de CoQ10 por gavagem oral uma vez ao dia), e o grupo 5 (CoQ10 + A $\beta$; recebeu CoQ10 por gavagem oral uma vez ao dia e injeções intraventriculares de $A \beta$ ), sendo a CoQ10 administrada por gavagem oral, uma vez ao dia, por 3 semanas antes e 3 semanas após a injeção de A . O estudo demonstrou resultados significativos com efeitos antioxidantes e antiapoptóticos, evidenciando assim, a redução dos efeitos de $A \beta$ e a neuroproteção contra efeitos prejudiciais da $\mathrm{A} \beta$ na plasticidade sináptica do hipocampo. 
A CoQ10 também demonstra efeitos significativos na ação antioxidante, podendo reduzir a deposição intracelular de A $\beta$ em modelos de camundongos tratados na administração de 1200 (mg/kg/dia) em duração de 60 dias (Yang et al., 2008 ; Yang et al., 2010).

Em estudo realizado por Wadsworth et al. (2008) onde foi realizado um ensaio clínico randomizado para receber dieta controle suplementado com CoQ com uma dose de $2 \mathrm{~g} / \mathrm{kg}$ de dieta em $10 \mathrm{~g} / \mathrm{kg}$ durante 30 dias com células de neuroblastoma MC65, foi demonstrado efeitos significativamente antioxidantes in vitro e no cérebro de camundongos. Ademais, em estudo de coorte com primeiro coorte com duração de 3 meses, e segundo coorte com duração de 5 meses em células de neuroblastoma de camundongo carregando AßPP com a mutação KM670 / 671NL, também foram obtidos efeitos significativamente antioxidantes pela diminuição de EO dos níveis cerebrais de carbonilas de proteínas, um marcador de EO e redução dos níveis de $\mathrm{A} \beta$ 42. Além disso, apresentou melhoria de desempenho cognitivo (Dumont et al., 2011).

Estudos mais recentes e ainda precoces sobre a CoQ10 com relação associada a DA vem sendo realizados na avaliação do efeito a curto prazo da CoQ10 aplicada topicamente na retina e na coróide na DA. Sendo assim, foi realizado um estudo clínico controlado randomizado em 62 pacientes com DA, em que foram executados exames neurológicos e oftalmológicos, incluindo tomografia de coerência óptica (OCT) para análise dos desfechos clínicos. Os resultados demonstraram melhoria na perda de células ganglionares retinais relacionadas à DA (Karakahya \& Özcan, 2020), à vista desse conhecimento, sabe-se que em doenças neurodegenerativas, como a DA, há a sensibilização da retina, vem sendo associados a efeitos nas reações bioquímicas celulares atribuídos aos níveis reduzidos de CoQ10 (Manzar et al., 2020).

Em terapias combinadas com outros tratamentos, como o uso da biotina com a CoQ10, há a demonstração de resultados significativos como neuroprotetora na DA (Attia et al., 2020). Além disso, o uso do ômega-3 associado a CoQ10 também demonstrou resultados significativos em efeitos neuroprotetores na DA, obtidos por mediação de atividades antioxidantes (Fouad, 2020). A terapia da CoQ10 associada ao ácido ascórbico também demonstrou efeito neuroprotetor na DA, atenuando o EO a longo prazo em camundongos (Frontiñán-Rubio et al., 2018).

Destarte, a CoQ10 parece ser um suplemento seguro com efeitos colaterais mínimos e baixo potencial de interação medicamentosa (Bonakdar \& Guarneri, 2005), demonstrando também, evidencias sobre sua segurança suplementada a longo prazo (Prangthip et al., 2016). Isto torna-se indispensável, uma vez que o tempo é considerado fator importante no uso de moléculas naturais, que geralmente não apresentam ações imediatas ou benefício concreto em curto período. Contudo, o uso constante por um longo período pode trazer vários benefícios em doenças neurodegenerativas (Ciulla et al., 2019). A segurança durante a gravidez e a lactação não foi bem estabelecido, entretanto a CoQ10 é aconselhável não ser tomado durante este período (Granick et al.,1996).

Além disso, há a necessidade de se atingir unanimidade com relação a sua dosagem ideal para o uso terapêutico pela disparidade de seus efeitos analisados e observadas em diversos estudos científicos (Gutierrez-Mariscal et al., 2020). Estudos farmacocinéticos em animais e humanos sugerem que a CoQ10 exógena não influencia a biossíntese de CoQ, e nem apresenta tendência em se acumular no plasma sanguíneo ou nos tecidos após a interrupção de sua suplementação (Hidaka et al., 2008).

Á vista disso, uma melhor compreensão acerca de efeito das modificações na alimentação sobre o metabolismo, a função mitocondrial e a produção de ROS durante os diferentes estágios da progressão da patologia são necessárias para o desenvolvimento estratégias terapêuticas seguras e eficazes para a DA (Tönnies \& Trushina, 2017), tornando assim cada vez mais relevante o entendimento e a compreensão do estado nutricional dos pacientes com a DA (Fernández \& Ribeiro, 2018). 
Research, Society and Development, v. 10, n. 2, e41210212751, 2021

(CC BY 4.0) | ISSN 2525-3409 | DOI: http://dx.doi.org/10.33448/rsd-v10i2.12751

\section{Considerações Finais}

A administração de CoQ10 na atenuação de respostas oxidativas de agregação $\beta$-Amiloide em modelos de DA demonstrou resultados significativos na diminuição de EO e ROS, impedindo assim, efeitos prejudiciais da $A \beta$ na plasticidade sináptica, obtendo efeito antiapoptótico e antinecrótica. Todavia, os resultados ainda são preliminares, visto que, ainda existem diversas lacunas.

Sendo assim, sugerimos a realização de mais estudos clínicos randomizados de controle em longo prazo, especialmente em humanos para melhor avaliação de sua eficácia e segurança, buscando estabelecer também parâmetros quanto a sua dosagem efetiva e segura visto a necessidade por novas terapias para a DA.

\section{Referências}

Attia, H., Albuhayri, S., Alaraidh, S., Alotaibi, A., Yacoub, H., Mohamad, R., \& Al-Amin, M. (2020). Biotin, coenzyme Q10, and their combination ameliorate aluminium chloride-induced Alzheimer's disease via attenuating neuroinflammation and improving brain insulin signaling. Journal of biochemical and molecular toxicology, e22519. Advance online publication.

Beal, M. F. (2004). Mitochondrial dysfunction and oxidative damage in Alzheimer's and Parkinson's diseases and coenzyme Q 10 as a potential treatment. Journal of bioenergetics and biomembranes, 36(4), 381-386.

Bezprozvanny, I., \& Mattson, M. P. (2008). Neuronal calcium mishandling and the pathogenesis of Alzheimer's disease. Trends in neurosciences, 31(9), 454463.

Bonakdar, R. A., \& Guarneri, E. (2005). Coenzyme Q10. American family physician, 72(6), 1065-1070.

Chen, Z., \& Zhong, C. (2014). Oxidative stress in Alzheimer's disease. Neuroscience bulletin, 30(2), $271-281$.

Choi, H., Park, H. H., Koh, S. H., Choi, N. Y., Yu, H. J., Park, J., Lee, Y. J., \& Lee, K. Y. (2012). Coenzyme Q10 protects against amyloid beta-induced neuronal cell death by inhibiting oxidative stress and activating the P13K pathway. Neurotoxicology, 33(1), 85-90.

Choi, J. H., Ryu, Y. W., \& Seo, J. H. (2005). Biotechnological production and applications of coenzyme Q 10. Applied microbiology and biotechnology, 68(1), 9-15.

Cioffi, F., Adam, R. H. I., \& Broersen, K. (2019). Molecular mechanisms and genetics of oxidative stress in Alzheimer's disease. Journal of Alzheimer's Disease, 1-37.

Ciulla, M., Marinelli, L., Cacciatore, I., \& Stefano, A. D. (2019). Role of Dietary Supplements in the Management of Parkinson's Disease. Biomolecules, 9(7), 271.

Darvesh, A. S., Carroll, R. T., Bishayee, A., Geldenhuys, W. J., \& Van der Schyf, C. J. (2010). Oxidative stress and Alzheimer's disease: dietary polyphenols as potential therapeutic agents. Expert review of neurotherapeutics, 10(5), 729-745.

De Felice, F. G., Velasco, P. T., Lambert, M. P., Viola, K., Fernandez, S. J., Ferreira, S. T., \& Klein, W. L. (2007). Abeta oligomers induce neuronal oxidative stress through an N-methyl-D-aspartate receptor-dependent mechanism that is blocked by the Alzheimer drug memantine. The Journal of biological chemistry, 282(15), 11590-11601.

Díaz-Casado, M. E., Quiles, J. L., Barriocanal-Casado, E., González-García, P., Battino, M., López, L. C., \& Varela-López, A. (2019). The Paradox of Coenzyme $\mathrm{Q}_{10}$ in Aging. Nutrients, 11(9), 2221.

Dumont, M., Kipiani, K., Yu, F., Wille, E., Katz, M., Calingasan, N. Y., Gouras, G. K., Lin, M. T., \& Beal, M. F. (2011). Coenzyme Q10 decreases amyloid pathology and improves behavior in a transgenic mouse model of Alzheimer's disease. Journal of Alzheimer's disease : JAD, 27(1), $211-223$.

Durán-Prado, M., Frontiñán, J., Santiago-Mora, R., Peinado, J. R., Parrado-Fernández, C., Gómez-Almagro, M. V., Moreno, M., López-Domínguez, J. A., Villalba, J. M., \& Alcaín, F. J. (2014). Coenzyme Q10 protects human endothelial cells from $\beta$-amyloid uptake and oxidative stress-induced injury. PloS one, 9(10), e109223.

Eratne, D., Loi, S. M., Farrand, S., Kelso, W., Velakoulis, D., \& Looi, J. C. (2018). Alzheimer's disease: clinical update on epidemiology, pathophysiology and diagnosis. Australasian psychiatry bulletin of Royal Australian and New Zealand College of Psychiatrists, 26(4), 347-357.

Farlow, M. R. (1998). Etiology and pathogenesis of Alzheimer's disease. American journal of health-system pharmacy, 55(2), S5-S10.

Fernández, S. S. M., \& Ribeiro, S. M. L. (2018). Nutrition and Alzheimer disease. Clinics in geriatric medicine, 34(4), 677-697.

Folkers, K. (1996). Relevance of the Biosynthesis of Coenzyme Q10and of the Four Bases of DNA as a Rationale for the Molecular Causes of Cancer and a Therapy. Biochemical and biophysical research communications, 224(2), 358-361.

Fouad, G. I. (2020). Combination of Omega 3 and Coenzyme Q10 Exerts Neuroprotective Potential Against Hypercholesterolemia-Induced Alzheimer's-Like Disease in Rats. Neurochemical Research, 1-14. 
Research, Society and Development, v. 10, n. 2, e41210212751, 2021

(CC BY 4.0) | ISSN 2525-3409 | DOI: http://dx.doi.org/10.33448/rsd-v10i2.12751

Frontiñán-Rubio, J., Sancho-Bielsa, F. J., Peinado, J. R., LaFerla, F. M., Giménez-Llort, L., Durán-Prado, M., \& Alcain, F. J. (2018). Sex-dependent cooccurrence of hypoxia and $\beta$-amyloid plaques in hippocampus and entorhinal cortex is reversed by long-term treatment with ubiquinol and ascorbic acid in the $3 \times$ Tg-AD mouse model of Alzheimer's disease. Molecular and cellular neurosciences, 92, 67-81.

Granick, B., Neubauer, D., \& Dermarderosian, A. (1996). The Lawrence review of natural products. St. Louis: Facts and Comparisons, 1-3.

Gustafson, D. R., Skoog, I., Rosengren, L., Zetterberg, H., \& Blennow, K. (2007). Cerebrospinal fluid beta-amyloid 1-42 concentration may predict cognitive decline in older women. Journal of neurology, neurosurgery, and psychiatry, 78(5), 461-464.

Gutierrez-Mariscal, F. M., Arenas-de Larriva, A. P., Limia-Perez, L., Romero-Cabrera, J. L., Yubero-Serrano, E. M., \& López-Miranda, J. (2020). Coenzyme $\mathrm{Q}_{10}$ Supplementation for the Reduction of Oxidative Stress: Clinical Implications in the Treatment of Chronic Diseases. International journal of molecular sciences, 21(21), 7870.

Hidaka, T., Fujii, K., Funahashi, I., Fukutomi, N., \& Hosoe, K. (2008). Safety assessment of coenzyme Q10 (CoQ10). BioFactors (Oxford, England), 32(1-4), 199-208.

Huang, X. G., Yee, B. K., Nag, S., Chan, S. T., \& Tang, F. (2003). Behavioral and neurochemical characterization of transgenic mice carrying the human presenilin-1 gene with or without the leucine-to-proline mutation at codon 235. Experimental neurology, 183(2), 673-681.

Kamat, P. K., Kalani, A., Rai, S., Swarnkar, S., Tota, S., Nath, C., \& Tyagi, N. (2016). Mechanism of Oxidative Stress and Synapse Dysfunction in the Pathogenesis of Alzheimer's Disease: Understanding the Therapeutics Strategies. Molecular neurobiology, 53(1), 648-661.

Kamat, P. K., Rai, S., Swarnkar, S., Shukla, R., Ali, S., Najmi, A. K., \& Nath, C. (2013). Okadaic acid-induced Tau phosphorylation in rat brain: role of NMDA receptor. Neuroscience, 238, 97-113.

Karakahya, R. H., \& Özcan, T. Ş. (2020). Salvage of the retinal ganglion cells in transition phase in Alzheimer's disease with topical coenzyme Q10: is it possible?. Graefe's Archive for Clinical and Experimental Ophthalmology, 258(2), 411-418.

Komaki, H., Faraji, N., Komaki, A., Shahidi, S., Etaee, F., Raoufi, S., \& Mirzaei, F. (2019). Investigation of protective effects of coenzyme Q10 on impaired synaptic plasticity in a male rat model of Alzheimer's disease. Brain research bulletin, 147, 14-21.

Lee, J., Boo, J. H., \& Ryu, H. (2009). The failure of mitochondria leads to neurodegeneration: Do mitochondria need a jump start?. Advanced drug delivery reviews, 61(14), 1316-1323.

Littarru, G. P. \& Tiano, L. (2010). Clinical aspects of coenzyme Q10: an update. Nutrition, 26(3), 250-254.

Lockwood, K., Moesgaard, S., \& Folkers, K. (1994). Partial and complete regression of breast cancer in patients in relation to dosage of coenzyme Q10. Biochemical and biophysical research communications, 199(3), 1504-1508.

López-Lluch, G., Del Pozo-Cruz, J., Sánchez-Cuesta, A., Cortés-Rodríguez, A. B., \& Navas, P. (2019). Bioavailability of coenzyme Q10 supplements depends on carrier lipids and solubilization. Nutrition (Burbank, Los Angeles County, Calif.), 57, 133-140.

Lovell, M. A., \& Markesbery, W. R. (2007). Oxidative damage in mild cognitive impairment and early Alzheimer's disease. Journal of neuroscience research, 85(14), 3036-3040.

Manzano-León, N., \& Mas-Oliva, J. (2006). Oxidative stress, $\beta$-amiloide peptide and Alzheimer’́s disease. Gaceta medica de Mexico, 142(3), $229-238$.

Manzar, H., Abdulhussein, D., Yap T. E., \& Cordeiro, M. F. (2020). Cellular Consequences of Coenzyme Q10 Deficiency in Neurodegeneration of the Retina and Brain. International Journal of Molecular Sciences, 21(23), 9299.

Organização Mundial De Saúde (OMS). Global Dementia Observatory. Organização Mundial De Saúde. 2017.

Pereira, A. S., Shitsuka, D. M., Parreira, F. J., \& Shitsuka, R. (2018). Metodologia da pesquisa científica.

Prangthip, P., Kettawan, A., Posuwan, J., Okuno, M., \& Okamoto, T. (2016). An Improvement of Oxidative Stress in Diabetic Rats by Ubiquinone-10 and Ubiquinol-10 and Bioavailability after Short- and Long-Term Coenzyme Q10 Supplementation. Journal of dietary supplements, 13(6), 647-659

Rai, S., Kamat, P. K., Nath, C., \& Shukla, R. (2014). Glial activation and post-synaptic neurotoxicity: the key events in Streptozotocin (ICV) induced memory impairment in rats. Pharmacology, biochemistry, and behavior, 117, 104-117.

Roberson, E. D., Halabisky, B., Yoo, J. W., Yao, J., Chin, J., Yan, F., Wu, T., Hamto, P., Devidze, N., Yu, G. Q., Palop, J. J., Noebels, J. L., \& Mucke, L. (2011). Amyloid-B/Fyn-induced synaptic, network, and cognitive impairments depend on tau levels in multiple mouse models of Alzheimer's disease. The Journal of neuroscience : the official journal of the Society for Neuroscience, 31(2), 700-711.

Sohal, R. S., \& Forster, M. J. (2007). Coenzyme Q, oxidative stress and aging. Mitochondrion, 7, S103-S111.

Tönnies, E., \& Trushina, E. (2017). Oxidative stress, synaptic dysfunction, and Alzheimer's disease. Journal of Alzheimer's Disease, 57(4), $1105-1121$.

Wadsworth, TL, Bishop, JA, Pappu, AS, Woltjer, RL, \& Quinn, JF (2008). Avaliação da coenzima Q como estratégia antioxidante na doença de Alzheimer. Journal of Alzheimer's disease: JAD , 14 (2), 225-234.

Wang, Z., Yang, L., \& Zheng, H. (2012). Role of APP and A $\beta$ in synaptic physiology. Current Alzheimer research, 9(2), 217-226. 
Research, Society and Development, v. 10, n. 2, e41210212751, 2021

(CC BY 4.0) | ISSN 2525-3409 | DOI: http://dx.doi.org/10.33448/rsd-v10i2.12751

Yang, X., Yang, Y., Li, G., Wang, J., \& Yang, E. S. (2008). Coenzyme Q10 attenuates beta-amyloid pathology in the aged transgenic mice with Alzheimer presenilin 1 mutation. Journal of molecular neuroscience: $M N$, 34(2), 165-171.

Yang, X., Dai, G., Li, G., \& Yang, E. S. (2010). Coenzyme Q10 reduces beta-amyloid plaque in an APP/PS1 transgenic mouse model of Alzheimer's disease. Journal of molecular neuroscience $M N, 41(1), 110-113$. 